\title{
Animal Versus Wind Dispersal and the Robustness of Tree Species to Deforestation
}

\section{Daniel Montoya, ${ }^{{ }^{*}}{ }^{*}$ Miguel A. Zavala, ${ }^{1,2}$ Miguel A. Rodríguez, ${ }^{1}$ Drew W. Purves ${ }^{3}$}

Studies suggest that populations of different species do not decline equally after habitat loss. However, empirical tests have been confined to fine spatiotemporal scales and have rarely included plants. Using data from 89,365 forest survey plots covering peninsular Spain, we explored, for each of 34 common tree species, the relationship between probability of occurrence and the local cover of remaining forest. Twenty-four species showed a significant negative response to forest loss, so that decreased forest cover had a negative effect on tree diversity, but the responses of individual species were highly variable. Animal-dispersed species were less vulnerable to forest loss, with six showing positive responses to decreased forest cover. The results imply that plant-animal interactions help prevent the collapse of forest communities that suffer habitat destruction.

$\mathrm{H}$ abitat destruction is often cited as the single greatest cause of global biodiversity loss (1). These anthropogenic changes trigger biological responses that sometimes end in a biotic collapse, a problem that has led ecologists to face the question of how much habitat is enough for species to persist (2). The dominant theoretical framework for understanding the effects of habitat loss is metapopulation theory, which focuses on the dynamic balance of local extinctions and colonizations that characterize fragmented populations at regional scales (3). According to this theory, regional habitat loss not only removes biodiversity held in the lost habitat but also reduces the occurrence of species within the remaining habitat (4). This idea has become a central tenet of conservation practice, causing a shift in focus from the local to the landscape scale. For example, it is the source of the current emphasis on the maintenance and creation of habitat corridors to foster dispersal among patches $(5,6)$. However, empirical tests of this prediction have been restricted to short-lived animal species [especially butterflies and birds $(7,8)]$, short spatial scales (9), and short time scales, over which observations are likely to be dominated by shortterm responses that may or may not be indicative of the long-term impacts of habitat loss.

We analyzed the relationship between local forest cover and the occurrence of 34 canopydominant tree species [ 28 native to the study region and 6 exotic (table S1)] in 89,365 survey sites distributed across peninsular Spain (10) (Fig. 1). The data set was extracted from the Spanish Second National Forest Inventory (IFN2), which placed a 25 -m-radius circular sample plot in each $1 \times 1 \mathrm{~km}$ grid cell that it classified as being

${ }^{1}$ Departamento de Ecología, Universidad de Alcalá, 28871 Alcalá de Henares, Madrid, Spain. ${ }^{2}$ Centro de Investigación Forestal, Instituto Nacional de Investigación y Tecnología Agraria y Alimentaria, Carretera de la Coruña km 7, 5 , 28040 Madrid, Spain. ${ }^{3}$ Microsoft Research Cambridge, 7 ]. J. Thomson Avenue, Cambridge CB3 OFB, UK.

*To whom correspondence should be addressed. E-mail: daniel.montoya@alu.uah.es forested [occupied by woody vegetation (11)]. For each plot $q$, we calculated a local forest cover $H_{q}$, defined as the fraction of the nearest eight grid cells to $q$ that were also classified as forested in IFN2 (using a larger neighborhood degraded the statistical significance of some effects documented here but had no qualitative effect on conclusions). Because the Iberian Peninsula has chronically suffered from forest destruction and conversion into agricultural and degraded states $(12), H_{q}$ is a measure of net forest loss from prehistory to the present. Thus, we interpret the species responses to $H_{q}$ observed in the IFN2 survey as responses to forest loss.

We used logistic regression to quantify, for each species $j$, the probability of occurrence of $j$ in plot $q$ as a function of $H_{q}$. For comparison among species we used the fitted logistic curves to calculate, for each species $j$, a scalar $\Omega_{j}$, defined as the natural log of the ratio of the probability of occurrence at $0 \%$ local forest cover to the probability of occurrence at $75 \%$ cover. Negative $\Omega_{j}$ implies that species $j$ shows a negative response to decreased forest cover and vice versa.
We used error propagation to calculate a conservative (upper) estimate of the confidence interval for $\Omega_{j}$. The results presented below are robust considering either native and exotic species combined or native species only [supporting online material (SOM)].

Of the 34 species, 24 showed a statistically significant negative response to decreased forest cover [negative $\Omega_{j}$ value with confidence intervals not including zero (Fig. 2A)]. This is consistent with the decrease in average tree species richness with decreased forest cover observed in the IFN2 data (Fig. 3) and in previous studies (13). The observed relationship between species richness in this case was approximately linear over most of the range in $H_{q}$, which was captured well by the logistic regressions (Fig. 3). However, richness was lower than expected for $H_{q} \geq 80 \%$ and $H_{q}=0$. Such abrupt changes could reflect the effects of spatial configuration (that is, fragmentation) when habitat cover goes from nearly continuous to fragmented (with the first appearance of edges) and falls to very low levels (14), although threshold responses can also result from some forms of animal-mediated dispersal (15).

Among species there was large and statistically significant variation in $\Omega_{j}$. For species with statistically significant negative $\Omega_{j}$ (those with confidence intervals not including zero), $\Omega_{j}$ ranged from -0.03 to -1.53 , which corresponds to a proportional reduction in probability of occurrence, for the 75 to $0 \%$ scenario, of 3 to $78 \%$. Moreover, there were six species with statistically significant positive responses to reductions in forest cover (Fig. 2A). These species were more likely to be found in plots surrounded by nonforested land.

If this magnitude of interspecific variation in response to forest loss proves to be typical, it will be critical to identify measurable species traits that predict it. Although we did not attempt an exhaustive search of such traits, we did examine the importance of two traits related to dispersal (seed size and animal- versus wind-mediated seed dis-

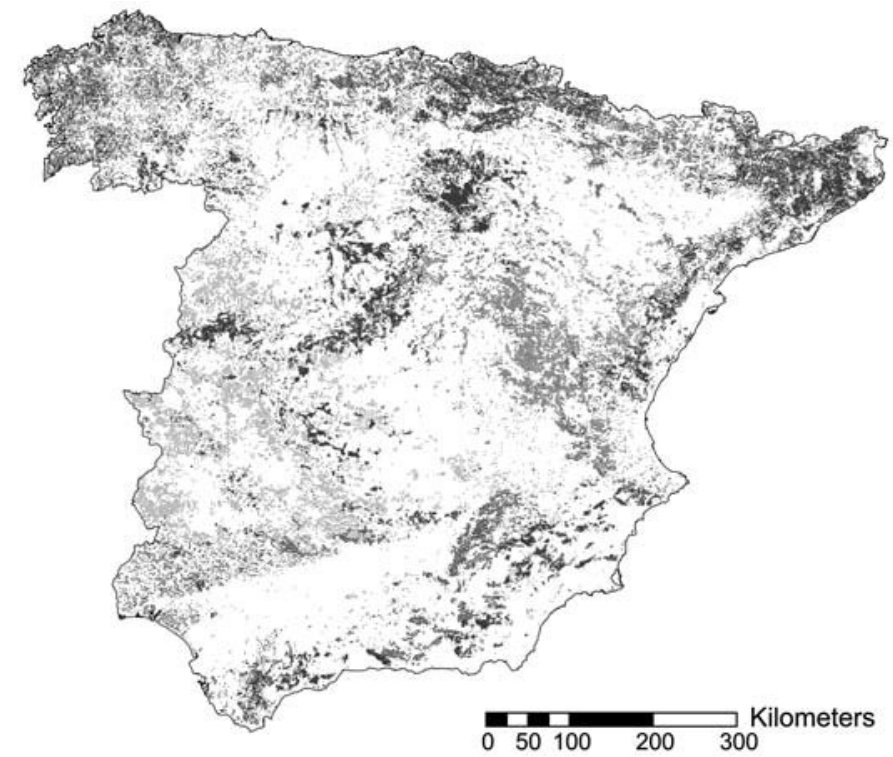

Fig. 1. Distribution of survey sites in peninsular Spain. IFN2 consisted of 89,365 circular sampling sites (radius $=25 \mathrm{~m}$ ) distributed across peninsular Spain (average density approximately one per square kilometer). Survey sites were placed in continuous forest locations, so their distribution matches that of the remaining forest. 
persal). Metapopulation theory has identified dispersal as crucial in determining species responses to habitat loss (16), and recent modifications of the Levins metapopulation model predict that animalmediated seed dispersal will confer increased species robustness to habitat loss (17). This is because, unlike wind, animals actively deliver seeds toward suitable patches (directed dispersal), and because some forms of animal dispersal increase the average dispersal distance (18). Both of these behaviors help keep physically isolated habitat patches demographically connected. Directed dispersal and long dispersal distances have been observed in our study region (19). Seed size affects dispersal distance (20) and is correlated with fecundity (21) and establishment probability (22), which are also highlighted as important by metapopulation theory.

We found that animal-dispersed species are, on average, less vulnerable to decreased forest cover than are wind-dispersed species (Fig. 2). The six species showing positive responses to deforestation were all animal-dispersed, and the two species with the largest negative response were wind-dispersed. To assess the possibility that the observed difference between the two groups (animal-and wind-dispersed) could have arisen by chance, we conducted a permutation test on the difference in the position of the groups in the list of species ranked by $\Omega_{j}$ : The probability of finding the observed difference was less than 0.005 (SOM).

The contrasted phylogenetic composition of wind- versus animal-dispersed species raises the possibility that phylogenetically conserved traits other than dispersal mode that are shared by closely related species caused the difference in response between animal- and wind-dispersed species (23). To examine this possibility, we used phylogenetic eigenvector regression [PVR (11)]. The proportion of variation of $\Omega_{j}$ that can be attributed to phylo- genetic relationships is low $\left(R^{2}=0.11\right)$. Moreover, an analysis of covariance (ANCOVA) including $\Omega_{j}$ as the response variable, dispersal mode as the explanatory variable, and the three main phylogenetic eigenvectors generated by PVR (which describe $92.5 \%$ of the phylogenentic structure in the data) as covariables still found significant differences in $\Omega_{j}$ between wind- and animal-dispersed species $(F=7.75890, P<0.05)$

In addition to the effects of forest cover, each tree species is likely to be affected by the pronounced gradients in climate, soil, and fire frequency observed in this region, and the interactions between all of these will need to be understood before any future species responses to changes in forest cover or climate can be predicted accurately. However, PCA showed no multicolinearity between forest cover and a set of 18 environmental variables that might be critical to plant growth, reproduction, and survival in Mediterranean and Atlantic systems (table S4). This shows that at the scale of peninsular Spain, local forest cover varies independently of climate, topography, soil, and major perturbation events, so that the simple logistic regression employed here would be expected to extract the correct average response to forest loss for each species. We also estimated $\Omega_{j}$ controlled for these environmental factors, using multiple logistic regression. The results support the conclusions of an overall negative, yet highly variable response to decreased forest cover among tree species, with some positive responses; and greater robustness of animaldispersed species (fig. S3, $P<0.005$ ).

Significant unexplained variation remains in the response of species within each dispersal group, but this is not surprising given the biological variation among species in either group. Just in terms of dispersal itself, both groups contain a large variation in seed size [although within either group we found no effect of seed weight on $\Omega_{j}$ (fig. S1)]; different

Fig. 2. (A) Sensitivity of 34 Spanish tree species to reduction in local forest cover $\left(\Omega_{j}\right)$, estimated using the maximum likelihood estimate of the parameters of a logistic regression relating the probability of occurrence of species $j$ to local forest cover. Gray arrows indicate non-native species. $\Omega_{j}$ is defined as the natural logarithm of the ratio of occurrence probabilities at 0 and $75 \%$ cover. Negative $\Omega_{j}$ implies a negative response to habitat loss. Species are ranked by $\Omega_{j \text {. }}$. Error bars are $95 \%$ confidence intervals on $\Omega_{j}$ calculated conservatively (16). (B) Average $\Omega_{j}$ of wind-dispersed species $(n=$ 12) and animal-dispersed

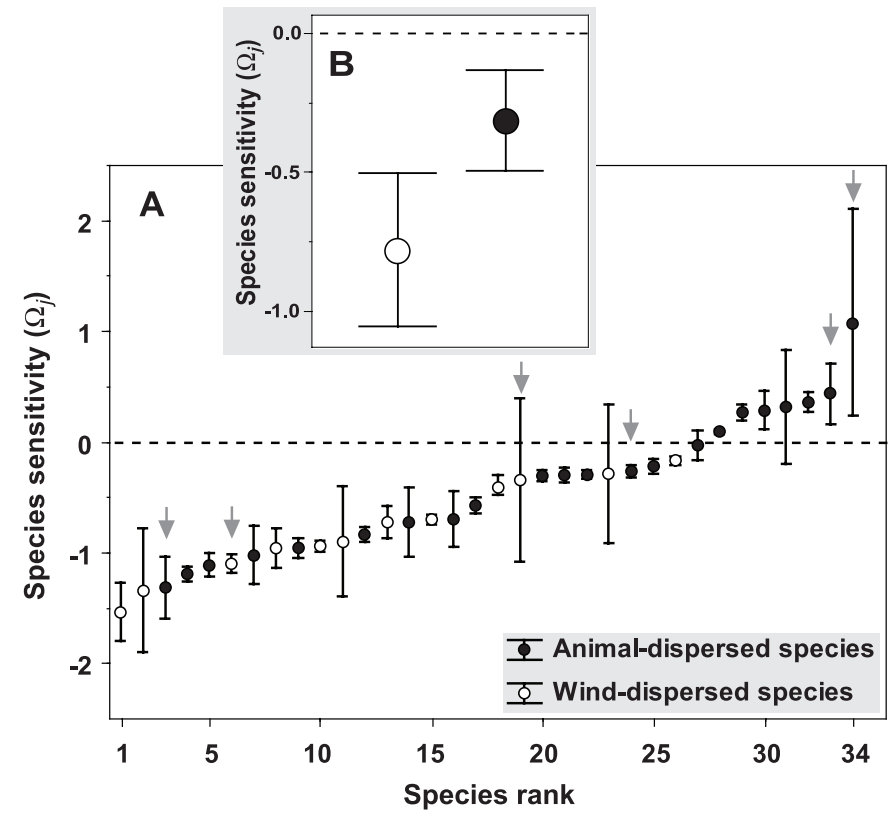

species $(n=22)$. Error bars are $95 \%$ confidence intervals on the mean $\Omega_{j}$ for each group. animal-dispersed species are dispersed by different combinations of birds and mammals [foraging behavior and body size differences among frugivores may affect seed dispersal distances $(24,25)]$; and many of the species are likely to benefit from occasional long-distance dispersal events by agents other than the dominant disperser (26). These species are also likely to differ in the other traits that metapopulation theory has predicted to be crucial in determining response to habitat loss (such as fecundity and local extinction rates). Nonetheless, our analysis suggests that the differences in species responses to local forest cover are to a large extent driven by the dispersal vector used by trees. This is consistent with predictions from metapopulation theory about the effects of animal-mediated directed dispersal. However, additional detailed field observations would be needed to rule out alternative explanations based on the interaction between dispersal and habitat loss. For example, lower amounts of habitat cover may be correlated with increased edge habitat, which has been observed in some cases to be preferred by seed-dispersing animals (27).

Whatever the mechanisms involved, the finding that animal-dispersed tree species are more robust to the effects of deforestation has an obvious implication for conservation policy: In the absence of detailed data (such as was available here), it might be expected that deforestation in other regions is more likely to threaten a given wind-dispersed, than a given animal-dispersed, plant species. However, the weight attached to this prediction should reflect the substantial withingroup variation in response documented here (Fig. 2) and the degree of extrapolation outside

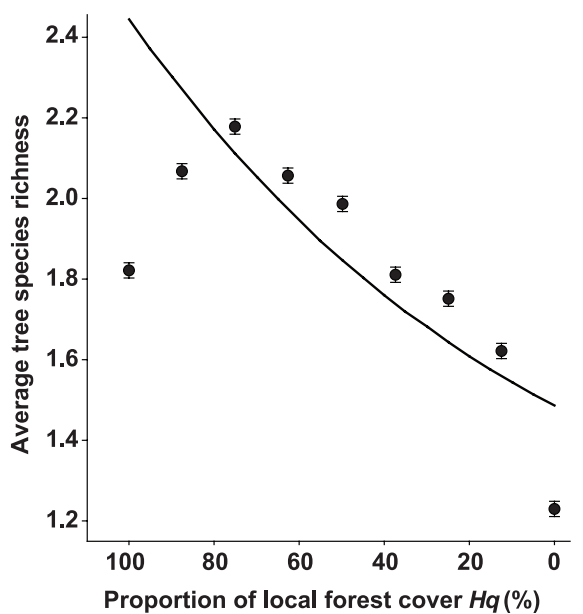

Fig. 3. Tree species richness (average number of species occurring in a 25 -m-radius circular plot) versus local forest cover $H_{q}$ : observed (points) and from the logistic regression (line, calculated by summing the predicted probability of occurrence over the 34 species). Error bars are standard errors on the observed average for each level of $H_{q}$. The observed richness is positively correlated with $H_{q i}$ that is, negatively correlated with forest loss (Spearman rank test, $\rho_{s}=0.73, P=0.038$ ). Species richness was calculated by referring to data for the 34 study species only. 
European temperate forests (such as to tropical forests or to plant species other than trees). Moreover, if seed-dispersing animals are as crucial to the persistence of plants as this and other studies suggest $(28,29)$, then the combination of habitat loss with direct and indirect removal of animals, to which many of the world's most diverse forests are subject, is likely to have more drastic effects than either perturbation alone. In these circumstances, animaldispersed species might be more, not less, sensitive to habitat loss. This points to the maintenance of the network of plant-animal interactions as a cornerstone of conservation policy and to the need for more studies of species responses to habitat loss.

\section{References and Notes}

1. R. V. Solé, J. Bascompte, Self Organization in Complex Ecosystems (Princeton Univ. Press, Princeton, N], 2007).

2. L. Fahrig, Biol. Conserv. 100, 65 (2001).

3. I. Hanski, Metapopulation Ecology (Oxford Univ. Press, Oxford, 1999).

4. M. Rees, R. Condit, M. Crawley, S. Pacala, S. Tilman, Science 293, 650 (2001)

5. E. I. Damschen, N. M. Haddad, ]. L. Orrock, J. J. Tewksbury, D. J. Levey, Science 313, 1284 (2006)
6. B. C.-L. Chetkiewicz, C. C. St. Clair, M. S. Boyce, Annu. Rev. Ecol. Syst. 37, 317 (2006)

7. L. Conradt, T. J. Roper, C. D. Thomas, Oikos 95, 416 (2001).

8. G. Ferraz et al., Science 315, 238 (2007).

9. I. Hanski, M. Kuussaari, M. Nieminen, Ecology 75, 747 (1994).

10. Segundo Inventario Forestal Nacional (Ministerio de Agricultura, Pesca y Alimentación, Madrid, 1995).

11. Information on materials and methods is available as supporting material on Science Online.

12. F. González Manzaneque, Bosques Ibéricos: Une Interpretación Geobotánica (Planeta, Barcelona, 2005).

13. J. Bascompte, M. A. Rodríguez, Ecol. Lett. 4, 417 (2001).

14. L. Fahrig, Annu. Rev. Ecol. Syst. 34, 487 (2003).

15. D. W. Purves, ]. Dushoff, J. Ecol. 93, 658 (2005).

16. I. Hanski, Nature 396, 41 (1998).

17. D. W. Purves, M. A. Zavala, K. Ogle, F. Prieto, J. M. Rey-Benayas, Ecol. Monogr. 77, 77 (2007).

18. K. Johst, R. Brandl, S. Eber, Oikos 98, 263 (2002).

19. ]. M. Gómez, Ecography 26, 573 (2003).

20. B. McEuen, L. M. Curran, Ecology 85, 507 (2004).

21. C. C. Smith, S. D. Fretwell, Am. Nat. 108, 499 (1974).

22. D. Sánchez-Gomez, F. Valladares, M. A. Zavala, New Phytol. 170, 795 (2006)

23. A. Purvis, P.-M. Agapow, ]. L. Gittleman, G. M. Mace, Science 288, 328 (2000)

24. O. Spiegel, R. Nathan, Ecol. Lett. 10, 718 (2007).

25. P. Jordano, C. García, J. A. Godoy, ]. L. García-Castaño, Proc. Natl. Acad. Sci. U.S.A. 104, 3278 (2007)
26. S. I. Higgins, R. Nathan, M. L. Cain, Ecology 84, 1945 (2003).

27. C. Restrepo, N. Gómez, S. Heredia, Ecology 80, 668 (1999).

28. J. M. Montoya, S. L. Pimm, R. V. Solé, Nature 442, 259 (2006).

29. ]. Bascompte, P. Jordano, Annu. Rev. Ecol. Syst. 38, 567 (2007)

30. We thank the Ministerio de Medio Ambiente, Spain; R. Vallejo and ]. A. Villanueva for help with IFN2 data; S. Pacala for hospitality during data preparation;

J. A. F. Diniz-Filho, L. M. Bini, and M. A. Olalla-Tárraga for help with phylogenetic analyses; and B. A. Hawkins, J. M. Montoya, D. Coomes, and ].-C. Svenning for comments on manuscripts. D.M. was supported by the Spanish Ministry for Education and Science (fellowship AP2004-0075).

M.A.R. and M.A.Z. were supported by the Spanish Ministry for Education and Science (grants CGL2006-03000/BOS and CGL2005-05830-C03-01/BOS, respectively).

\section{Supporting Online Material}

www.sciencemag.org/cgi/content/full/1158404/DC1

Materials and Methods

Figs. S1 to S3

Tables. S1 to S4

References

28 March 2008; accepted 15 May 2008

Published online 5 June 2008;

10.1126/science. 1158404

Include this information when citing this paper. 


\section{Science \\ МIAAAS}

\section{Supporting Online Material for}

\section{Animal Versus Wind Dispersal and the Robustness of Tree Species to Deforestation}

Daniel Montoya,* Miguel A. Zavala, Miguel A. Rodríguez, Drew W. Purves

*To whom correspondence should be addressed. E-mail: daniel.montoya@alu.uah.es

Published 5 June 2008 on Science Express

DOI: $10.1126 /$ science.1158404

This PDF file includes:

Materials and Methods

Tables S1 to S4

Figs. S1 to S3

References 
2

\section{SUPPORTING ONLINE MATERIAL}

\section{Material and Methods}

\section{Region and dataset}

The Iberian Peninsula spans from $36^{\circ} \mathrm{N}$ to $43.5^{\circ} \mathrm{N}$ and $9^{\circ} \mathrm{W}$ to $3^{\circ} \mathrm{E}$, housing a large altitudinal gradient (sea level - 3500 meters); it comprises a mosaic of different climates (from semiarid to Mediterranean and humid Atlantic climates), and a number of quite different landscapes such as natural and managed forests, savannas, shrublands, grasslands, agriculture fields and urban areas. This region is located in a known biodiversity hot-spot (Mediterranean basin; S1). The Second Spanish Forest Inventory (IFN2, 1986-1996; ICONA 1995) is an extensive dataset consisting of 91,200 circular sample plots distributed across the currently-wooded surface of peninsular Spain, with an approximate density of one site per $1 \mathrm{Km}^{2}$. Each sampling site was located in the field using pre-generated UTM coordinates, and was sampled for many attributes, including presence/absence of several species of trees; stem diameter and height for trees of some species; altitude and slope. The IFN2 assigns a code for each tree species or group of species. Because codes related to multiple species do not distinguish individual species, only species with specific codes (codes referring to single species) were considered here. For the purpose of this study, we extracted the presence/absence data for 34 canopy-dominant tree species commonly found in Mediterranean and Atlantic forests of the study region (where presence or absence means presence or absence of a living tree of the species within a circular survey plot with $25 \mathrm{~m}$ radius). These species comprise a wide range of niches and biological traits (Table S1). Tree species with a main distinctive dispersal mode (wind / animal) were studied (S2- 4); species with mixed dispersal (wind + animal) were excluded from the analyses (i.e. Pinus pinea). Sampling sites with georreferencing mismatches and errors in the climate variable estimations were excluded. This left 89,365 sites and 34 species in the analysis.

The IFN2 includes 6 exotic species that naturally invaded forest systems in the study region. These species were considered in our analyses provided they had a clear distinctive dispersal mode. In addition, part of the distribution area of some exotic species surveyed in the IFN2 consists of plantations. But importantly, considering only native species, or both native and exotic species, had no material impact on conclusions (Figure S2).

Since the survey sites were only placed in $1 \times 1 \mathrm{~km}$ grid-cells currently occupied by forest (defined as dominated by woody plants), an estimate of the proportion of forest habitat cover could be estimated as FS/NoFS, where FS (Forest Sites) is the number of surveyed sites (those where trees are present) in a given neighbourhood around a focal site, and NoFS (Non-Forest Sites) is the number of sites within that neighbourhood that were not surveyed (those not dominated by woody plants). Thus, we generated a continuous $1 \mathrm{Km}$ resolution grid covering peninsular Spain to which the survey network was superimposed. Points in this grid that matched the survey sites were defined as forest sites; conversely, non-matching points were defined as non-forest sites. 'Forest cover' measures the continuity of forest by looking at neighbour sites (corresponding to areas of $8 \mathrm{Km}^{2}$ around each site), giving an estimation of habitat cover surface ranging from $0 \%$ to $100 \%$. 
To avoid linearity assumptions in the relationship between species' occurrence probability (which is bounded 0-1) and the proportion of forest cover, we fitted logistic regression models to estimate the probability of occurrence of each species in each site.

$$
P\left(j, H_{q}\right)=\frac{e^{-K\left(j, H_{q}\right)}}{1+e^{-K\left(j, H_{q}\right)}}
$$

$$
K\left(j, H_{q}\right)=M_{j}+C_{j}\left(1-H_{q}\right)
$$

Where $P\left(j, H_{q}\right)$ is the probability of finding species $j$ in site $q$ with local habitat cover $H_{q}$, and $M_{j}$ and $C_{j}$ are species-specific parameters. The logistic regression returned maximum likelihood estimates (MLE) for $M_{j}$ and $C_{j}$, together with 95\% intervals on each parameter. For each species, the regression was performed using 89,365 $\mathrm{H}_{q}, \mathrm{O}_{j, q}$ pairs, where $O_{j, q}$ is the observed occupancy of species $j$ in plot $q$ (1 present, 0 absent). The regression treated these data as independent, and so did not include the potential effects of spatial autocorrelation, i.e. a tendency for plots close to each other to have similar $H_{q}$, and / or $O_{j, q}$, values. In reality this autocorrelation reduces the effective sample size of the regression, compared to that carried out here, by a fraction that is currently unknown; although the effects of this are unlikely to be substantial here because the (uncorrected) sample size is so large (89,365 for each species) compared to the number of parameters to be estimated ( 2 for each species), and because the standard deviation on parameter estimates shows only a square-root relationship to sample size. Moreover, we used a highly conservative method to estimate the confidence intervals on $\Omega_{j}$ (see below): this method is likely to have increased the confidence intervals by a larger fraction than would the inclusion of the effects of autocorrelation.

For each species $j$, the parameter estimates corresponded to a continuous function relating habitat cover and occurrence, and the uncertainty in parameters corresponded to uncertainty in this function. To facilitate comparison among species, we needed, from these continuous functions, to generate a scalar metric measuring sensitivity to forest loss. We chose to use the proportional reduction in occurrence caused by a reduction in forest cover from a high value (0.75) to zero:

$$
\Omega_{j}=\ln \left\{P\left(j, H_{q}=0\right) / P\left(j, H_{q}=0.75\right)\right\}
$$

Where $P\left(j, H_{q}\right)$ is calculated using the MLE estimates for the parameters $M_{j}$ and $C_{j}$. Note that a negative $\Omega_{j}$ implies a negative response of occurrence to reduced local forest cover. Also, the logarithmic scale makes the relationship between $\Omega_{j}$ and the proportional reduction in occurrence non-linear: for example, $\Omega_{j}=-2$ implies an $86 \%$ reduction in occurrence, -1 implies $63 \%$ reduction, -0.5 implies $40 \%$ reduction, and +1 implies a $170 \%$ increase in occurrence.

To produce confidence intervals on $\Omega_{j}$ for each species, we used the following simple error propagation approach: (1) extract, for $M_{j}$ and $C_{j}$, the lower and upper $95 \%$ confidence interval; (2) generate the four combinations of $M_{j}$ and $C_{j}$ that can be given by combining these lower and upper estimates; (3) apply eq. S3 using $P\left(j, H_{q}\right)$ calculated from each of the four combinations; (4) report the highest and lowest $\Omega_{j}$ values from the four calculated values. This method allows for independent variation in 
$97 M_{j}$ and $C_{j}$, and allows for both parameters to be simultaneously at the edge of their 5\%

98 confidence interval. It is therefore a conservative method compared to a formal error

99 propagation: i.e., the reported intervals are larger than those that would have been

100 produced from formal error propagation.

101

102

103

104

105

106

107

108

109

110

111

112

113

114

115

116

117

118

119

120

121

122

123

124

125

126

127

128

129

130

131

132

133

134

135

136

137

138

139

140

141

142

143

144

145

\section{Permutation Test}

We ranked species $\Omega_{j}$ (species 1 , species2,..., species 34), so the average position of an animal- and wind-dispersed species in that list could be obtained. The difference between the average position numbers was used as a test statistic to evaluate the probability of obtaining, by chance, the observed difference in average rank between wind- and animal-dispersed species. 10,000 sets of random species ranks were produced, where each species was assigned a rank (1-34) at random. For each of these 10,000 sets, the difference between the average rank for animal vs wind-dispersed species was calculated. This yielded a frequency distribution of the difference between the groups, corresponding to a null hypothesis of no true difference in $\Omega_{j}$ between the groups. We then noted where the observed value of the difference between the groups lay in the frequency distribution, which corresponded to the probability of obtaining the observed difference without a true difference between the groups.

\section{Phylogenetic analyses}

The amount of phylogenetic signal in $\Omega_{j}$ was quantified with Phylogenetic eigenVector Regression (PVR; S5). This method tests whether species traits (in this case, $\boldsymbol{\Omega}_{j}$ ) are significantly associated with phylogeny, partitioning the phenotypic variation in a certain trait into phylogenetic (among lineages, $\mathbf{P}$ ) and nonphylogenetic or specific (within species, $\mathbf{S}$ ) components, based on multivariate analysis $\left(\Omega_{j}=\mathbf{P}+\mathbf{S}\right)$. The idea is that phylogeny can be expressed as a set of orthogonal vectors obtained by an eigenanalysis of a phylogenetic distance matrix. These vectors can then be used as predictors of $\Omega_{j}$ in any form of linear or non-linear modelling.

The starting point is the construction of the phylogenetic distance matrix $\mathbf{D}$, for which we used the phylogeny for existing seed-plant families hosted in the Webpage of the Missouri Botanical Garden, and that has been compiled and is being continuously updated by P.F. Stevens using different sources (the phylogeny and details about its construction are available at: www.mobot.org/MOBOT/research/APweb/) (accessed, December 2007). For each possible pair of species in $\mathbf{D}$, we considered that their phylogenetic distance was the minimum number of nodes connecting them in the phylogeny. Then, we used principal coordinates analysis (PCoA) to extract eigenvalues and eigenvectors from this matrix after performing a double-center transformation (the row and column means were substracted from each element and the grand mean was added, after transforming original distances into $-0.5 D_{i j}^{2}$ ). We used the coefficients of the eigenvectors of $\mathbf{D}$ as the coordinates of the species in the reduced multivariate space expressing variation among them in the hyperspace defined by phylogenetic relatedness (numerical details about PVR calculations are provided in S5).

The first three eigenvectors from the PVR explained most of the phylogenetic structure existing in $\mathbf{D}$ (Table S2), and were identified as capturing meaningful phylogenetic relationships by a scree-plot +1 procedure, a conservative method of eigenvector selection (S6). Accordingly, to test whether there were differences between wind- and animal-dispersed species in terms of $\boldsymbol{\Omega}_{j}$ that are independent of phylogenetic 
146 inertia, we included these eigenvectors as covariables in an analysis of covariance

147 (ANCOVA) in which the response and explanatory variables were $\Omega_{j}$ and dispersal

148 mode, respectively. The two groups (animal dispersed, wind dispersed) showed

149 significantly different mean $\Omega_{j}$ values, either when considering all 34 species, or when

150 analyzing only the 28 species that are native to the study region (Table S3a, b).

151 Therefore, we concluded that the difference in average $\Omega_{j}$ between wind-dispersed and

152 animal-dispersed species was not caused by phylogenetic autocorrelation.

153

154 Multicollinearity

155 In addition to the effects of habitat cover, each tree species is likely to be affected by the

156 gradients in climate, soil and fire frequency occurring in the study region, raising the

157 possibility that apparent responses to habitat cover (and any apparent differences in

158 response among species) are driven by environmental factors correlated with habitat

159 cover (multicollinearity). This problem occurs in any study of species occurrence vs.

160 habitat cover, except in the rare cases where the habitat gradient has been exposed

161 experimentally. To assess the sensitivity of our conclusions to this problem, we first

162 extracted, for each survey site, a set of 18 climatic, topographic and soil variables (listed

163 in Table S4) which might be critical to plant physiological function and survival in the

164 Mediterranean and Atlantic systems. Principal component analysis showed no

165 multicollinearity between forest cover and these variables (Table S4). This showed that,

166 at the scale of peninsular Spain, forest cover varies almost independently of climate,

167 topography, soil, and major perturbation events (Figure S3), making mutlicollineariy an

168 unlikely explanation of the results.

169 As a additional check, we performed combined species-specific logistic regression that

170 included included environmental variables (the axis scores from the PCA described

171 above) and forest cover, in order to extract estimates for $\boldsymbol{\Omega}_{j}$ controlled for

172 environmental factors (Fig. S3). The pattern of interspecific variation in $\Omega_{j}$ was not

173 qualitatively altered by including environmental variables (compare Fig S3 with Fig. 2),

174 and the difference in $\Omega_{j}$ between animal- and wind-dispersed species remained

175 statistically significant (permutation test: $p<0.005$ ). 
176

177

178

179

180

181

182

183

184

185

186

187

188

189

\section{SUPPLEMENTARY REFERENCES}

S1. N. Myers, R. A. Mittermeier, C. G. Mittermeier, G. A. da Fonseca, J. Kent, Nature 403, 853-858 (2000)

S2. González Manzaneque, F. (Ed.), Bosques Ibéricos: Una Interpretación Geobotánica (Planeta, Barcelona, 2005)

S3. Catalán Bachiller, G., Semillas de Árboles y Arbustos Forestales (ICONA, Madrid, 1991)

S4. López González, G., Guía Incafo de los Árboles y Arbustos de la Península Ibérica (Incafo, Madrid, 1994)

S5. $\quad$ Diniz-Filho, J. A. F., de Sant'Ana, C. E. R., Bini, L. M., Evolution 52(5), 12471262 (1998)

S6. Jackson, D. A., Ecology 74(8), 2204-2214 (1993) 
Table S1.

191

\begin{tabular}{|l|l|r|r|r|}
\hline Species & Dispersal mode & \multicolumn{1}{|c|}{$M_{j}$} & \multicolumn{1}{|c|}{$C_{j}$} & \multicolumn{1}{|c|}{$\Omega_{j}$} \\
\hline Populus tremula & Wind & 4.572 & 2.054 & -1.53 \\
Picea abies & Wind & 6.202 & 1.78 & -1.33 \\
Quercus borealis & Animal & 4.83 & 1.763 & -1.32 \\
Fagus sylvatica & Animal & 1.821 & 1.728 & -1.22 \\
Phyllirea latifolia & Animal & 2.977 & 1.523 & -1.12 \\
Pinus radiata & Wind & 2.434 & 1.542 & -1.18 \\
Quercus canariensis & Animal & 4.873 & 1.363 & -1.02 \\
Pinus sylvestris & Wind & 1.099 & 1.498 & -0.99 \\
Quercus petraea & Animal & 2.696 & 1.325 & -0.96 \\
Abies alba & Wind & 4.108 & 1.288 & -0.96 \\
Populus alba & Wind & 7.455 & 1.198 & -0.90 \\
Ilex aquifolium & Animal & 2.202 & 1.192 & -0.85 \\
Pinus pinaster & Wind & 0.918 & 1.175 & -0.74 \\
Pinus uncinata & Wind & 3.866 & 0.981 & -0.73 \\
Taxus baccata & Animal & 5.458 & 0.967 & -0.72 \\
Malus sylvestris & Animal & 5.019 & 0.929 & -0.69 \\
Corylus avellana & Animal & 2.578 & 0.804 & -0.58 \\
Pinus nigra & Wind & 1.806 & 0.643 & -0.43 \\
Ailanthus altissima & Wind & 7.41 & 0.456 & -0.34 \\
Quercus robur & Animal & 1.827 & 0.456 & -0.31 \\
Quercus faginea & Animal & 1.448 & 0.471 & -0.30 \\
Quercus suber & Animal & 2.581 & 0.419 & -0.30 \\
Abies pinsapo & Wind & 7.129 & 0.376 & -0.28 \\
Castanea sativa & Animal & 2.337 & 0.383 & -0.27 \\
Rhamnus alaternus & Animal & 2.766 & 0.307 & -0.22 \\
Pinus halepensis & Wind & 1.516 & 0.274 & -0.17 \\
Frangula alnus & Animal & 4.251 & 0.037 & -0.03 \\
Quercus ilex & Animal & 3.51 & -0.202 & 0.09 \\
Olea europaea & Animal & 3.293 & -0.375 & 0.27 \\
Ceratonia siliqua & Animal & 7.954 & -0.39 & 0.29 \\
Celtis australis & Animal & -0.423 & 0.32 \\
Juniperus thurifera & Animal & -0.501 & 0.36 \\
Juglans regia & Animal & -1.722 & 0.787 \\
Rhus coriaria & & & \\
\hline
\end{tabular}

192

193

194 List of tree species included in the analysis. Dispersal mode is indicated. Parameter

195 estimates of the logistic regression models $\left(M_{j}, C_{j}\right)$ are provided for each species,

196 together with $\Omega_{j}$ calculated at the MLE estimates for $M_{j}$ and $C_{j}$. Species are given in

197 rank order of $\Omega_{j}$ (a negative $\Omega_{j}$ implies a negative response to forest loss). Exotic

198 species are highlighted in bold. 
Table S2.

200

201

\begin{tabular}{|c|c|c|c|}
\hline Eigenvector i & Eigenvalue & $\mathrm{R}^{2}$ & Cumulative $\mathrm{R}^{2}$ \\
\hline 1 & 5993.082637 & 79.3794 & 79.3794 \\
\hline 2 & 678.8968592 & 8.9921 & 88.3715 \\
\hline 3 & 310.1979661 & 4.1086 & 92.4801 \\
\hline 4 & 210.6672177 & 2.7903 & 95.2705 \\
\hline 5 & 94.52496831 & 1.252 & 96.5225 \\
\hline 6 & 79.52899032 & 1.0534 & 97.5758 \\
\hline 7 & 72.2764274 & 0.9573 & 98.5332 \\
\hline 8 & 55.34903771 & 0.7331 & 99.2663 \\
\hline 9 & 49.02118729 & 0.6493 & 99.9156 \\
\hline 10 & 40.93208026 & 0.5422 & 100 \\
\hline 11 & 30.89333637 & 0.4092 & 100 \\
\hline 12 & 18 & 0.2384 & 100 \\
\hline 13 & 1.69103547 & 0.0224 & 100 \\
\hline 14 & 0.5 & 0.0066 & 100 \\
\hline 15 & 0.5 & 0.0066 & 100 \\
\hline 16 & 0.5 & 0.0066 & 100 \\
\hline 17 & 0.5 & 0.0066 & 100 \\
\hline 18 & 0.5 & 0.0066 & 100 \\
\hline 19 & 0.5 & 0.0066 & 100 \\
\hline 20 & 0.5 & 0.0066 & 100 \\
\hline 21 & 0.5 & 0.0066 & 100 \\
\hline 22 & 0.5 & 0.0066 & 100 \\
\hline 23 & 0.5 & 0.0066 & 100 \\
\hline 24 & 0.5 & 0.0066 & 100 \\
\hline 25 & 0.5 & 0.0066 & 100 \\
\hline 26 & 0.5 & 0.0066 & 100 \\
\hline 27 & 0.5 & 0.0066 & 100 \\
\hline 28 & 0.5 & 0.0066 & 100 \\
\hline 29 & 0.5 & 0.0066 & 100 \\
\hline 30 & 0.0 & 0.0 & 100 \\
\hline 31 & -3.48786972 & -0.0462 & 100 \\
\hline 32 & -4.87072252 & -0.0645 & 100 \\
\hline 33 & -25.88538769 & -0.3429 & 100 \\
\hline 34 & -60.89671049 & -0.8066 & 100 \\
\hline
\end{tabular}

204 Eigenvectors generated by the eigenanalysis of the phylogenetic distance matrix D 205 among tree species. The first three eigenvectors explained 92.5\% of the phylogenetic 206 signal and are emphasized in grey. These eigenvectors were identified by the scree-plot $207+1$ method as the most meaningful ones (6) and used as covariables in ANCOVA 208 analysis. 
Table S3.

210

211 A

\begin{tabular}{lccccc}
\multicolumn{1}{c}{$\mathbf{N}=\mathbf{3 4}$} & SS & df & MS & F & p \\
\hline Intercept & 17.39162 & 1 & 17.39162 & 31.21513 & 0.000005 \\
Eigenvector 1 & 0.50904 & 1 & 0.50904 & 0.91365 & 0.347048 \\
Eigenvector 2 & 0.00987 & 1 & 0.00987 & 0.01771 & 0.895042 \\
Eigenvector 3 & 3.18965 & 1 & 3.18965 & 5.72490 & 0.023425 \\
Dispersal & 4.32290 & 1 & 4.32290 & $\mathbf{7 . 7 5 8 9 0}$ & $\mathbf{0 . 0 0 9 3 2 1}$ \\
Error & 16.15745 & 29 & 0.55715 & &
\end{tabular}

212

213

214

B

\begin{tabular}{lccccc}
\multicolumn{1}{c}{$\mathbf{N}=\mathbf{2 8}$} & SS & df & MS & F & p \\
\hline Intercept & 16.59317 & 1 & 16.59317 & 38.70054 & 0.000002 \\
Eigenvector 1 & 0.86676 & 1 & 0.86676 & 2.02157 & 0.168498 \\
Eigenvector 2 & 0.02190 & 1 & 0.02190 & 0.05107 & 0.823208 \\
Eigenvector 3 & 0.81040 & 1 & 0.81040 & 1.89012 & 0.182435 \\
Dispersal & 3.15059 & 1 & 3.15059 & $\mathbf{7 . 3 4 8 1 7}$ & $\mathbf{0 . 0 1 2 4 7 1}$ \\
Error & 9.86144 & 23 & 0.42876 & &
\end{tabular}

217 Phylogenetically-controlled analysis of covariance (ANCOVA) on $\boldsymbol{\Omega}_{\mathrm{j}}$. The first 3

218 eigenvectors generated by the eigenanalysis of the phylogenetic distance matrix were

219 used as covariates. (A) Results for native and exotic species $(\mathrm{N}=34)$. (B) Results for 220 native species only $(\mathrm{N}=28)$. ( $\mathrm{SS}=$ Sum of squares; $\mathrm{df}=$ Degrees of freedom; $\mathrm{MS}=$ 221 Mean of squares). 


\begin{tabular}{lcccc}
\multicolumn{1}{c}{ Variables } & Factor $\mathbf{1}$ & Factor 2 & Factor 3 & Factor 4 \\
\hline Annual Precipitation & $\mathbf{0 . 7 4 6 7 7 9}$ & -0.552574 & 0.116271 & 0.052051 \\
Spring Precipitation & $\mathbf{0 . 7 9 7 2 8 1}$ & -0.470433 & 0.117155 & 0.049064 \\
Summer Precipitation & $\mathbf{0 . 8 4 4 8 8 8}$ & -0.027751 & 0.022345 & 0.007831 \\
Potential Evapotranspiration & $\mathbf{- 0 . 6 6 1 2 2 5}$ & -0.327717 & -0.049185 & 0.075896 \\
(Thornwaite's formula) & & & & \\
Annual Hydric Index & -0.285126 & -0.421425 & 0.113343 & 0.375789 \\
Drougth Length & $\mathbf{- 0 . 6 8 1 7 2 0}$ & 0.238016 & -0.064637 & -0.520283 \\
Drougth Intensity & -0.541502 & 0.159017 & -0.038245 & $\mathbf{- 0 . 6 4 7 1 8 0}$ \\
Mean Annual Temperature & $\mathbf{- 0 . 8 2 6 8 6 4}$ & -0.502501 & -0.139506 & 0.055236 \\
Potential Evapotranspiration & $\mathbf{- 0 . 9 3 4 1 7 9}$ & -0.231905 & -0.150795 & 0.109854 \\
(Jensen \& Haise formula) & & & & \\
Annual Radiation & $\mathbf{- 0 . 8 1 9 7 8 7}$ & 0.301444 & -0.143696 & 0.176955 \\
Mean Temperature Warmest & $\mathbf{- 0 . 9 2 7 5 1 0}$ & -0.084287 & -0.170067 & 0.194822 \\
Month & & & & \\
Mean Temperature Coldest & -0.573380 & $\mathbf{- 0 . 7 5 6 8 0 9}$ & -0.099752 & -0.073076 \\
Month & & & & \\
Termal Oscillation & -0.535972 & $\mathbf{0 . 6 5 0 8 4 2}$ & -0.103618 & 0.304678 \\
Altitude & 0.362111 & $\mathbf{0 . 8 0 5 5 1 2}$ & 0.018220 & 0.072400 \\
Aspect & -0.100998 & 0.008870 & -0.106684 & 0.163563 \\
Slope & 0.405246 & 0.160215 & -0.131351 & 0.095624 \\
Soil & 0.049918 & -0.182366 & 0.177293 & -0.434954 \\
Annual Fire Rate & 0.158481 & -0.553708 & 0.115610 & -0.112220 \\
Forest Cover $\left(H_{q}\right)$ & -0.301332 & 0.028006 & $\mathbf{0 . 8 4 8 3 1 6}$ & 0.081156 \\
\hline
\end{tabular}

List of environmental variables included in the principal component analysis (PCA).

228 The list includes climatic, topographic, soil and disturbance variables that the authors

229 considered might be important to tree physiological function and survival in

230 Mediterranean and Atlantic ecosystems. Each variable was estimated for each survey

231 plot. The PCA shows that local forest habitat cover $\left(H_{q}\right)$ is an independent factor not

232 related to climate, topography, soil or fire disturbance (factor 3). 
233

234

235

236

237

238

Figure S1. Sensitivity of tree species to reductions in forest cover $\left(\Omega_{j}\right)$ as a function of seed size. Species are ranked by $\Omega_{j}$. Bubble size is proportional to seed weight. White symbols are animal-dispersed species; gray symbols are wind-dispersed species. Only 23 species are shown, because seed weights were not available for the other species.

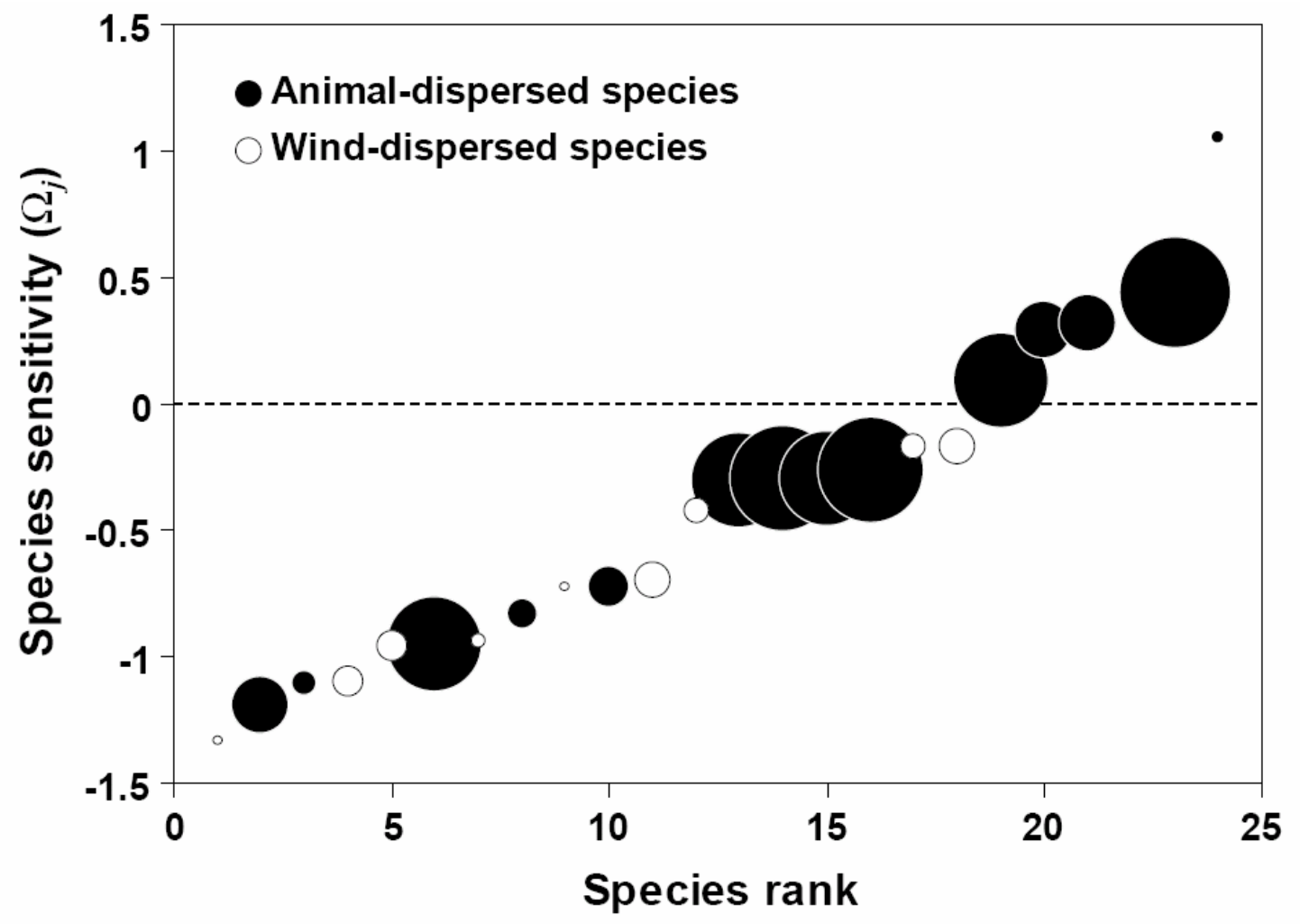


Figure S2. (A) Sensitivity of tree species to forest habitat loss $\Omega_{j}$ (at $1 \mathrm{Km}$ ) for native species only $(\mathrm{N}=28)$. Results from permutation test for average difference between animal- and wind-dispersed species: $\mathrm{p}<0.05$. (B) Average $\Omega_{j}$ for native wind-dispersed species $(n=10)$ and native animal-dispersed species $(n=18)$. Error bars are $95 \%$ confidence intervals on the mean $\Omega_{j}$ for each group.

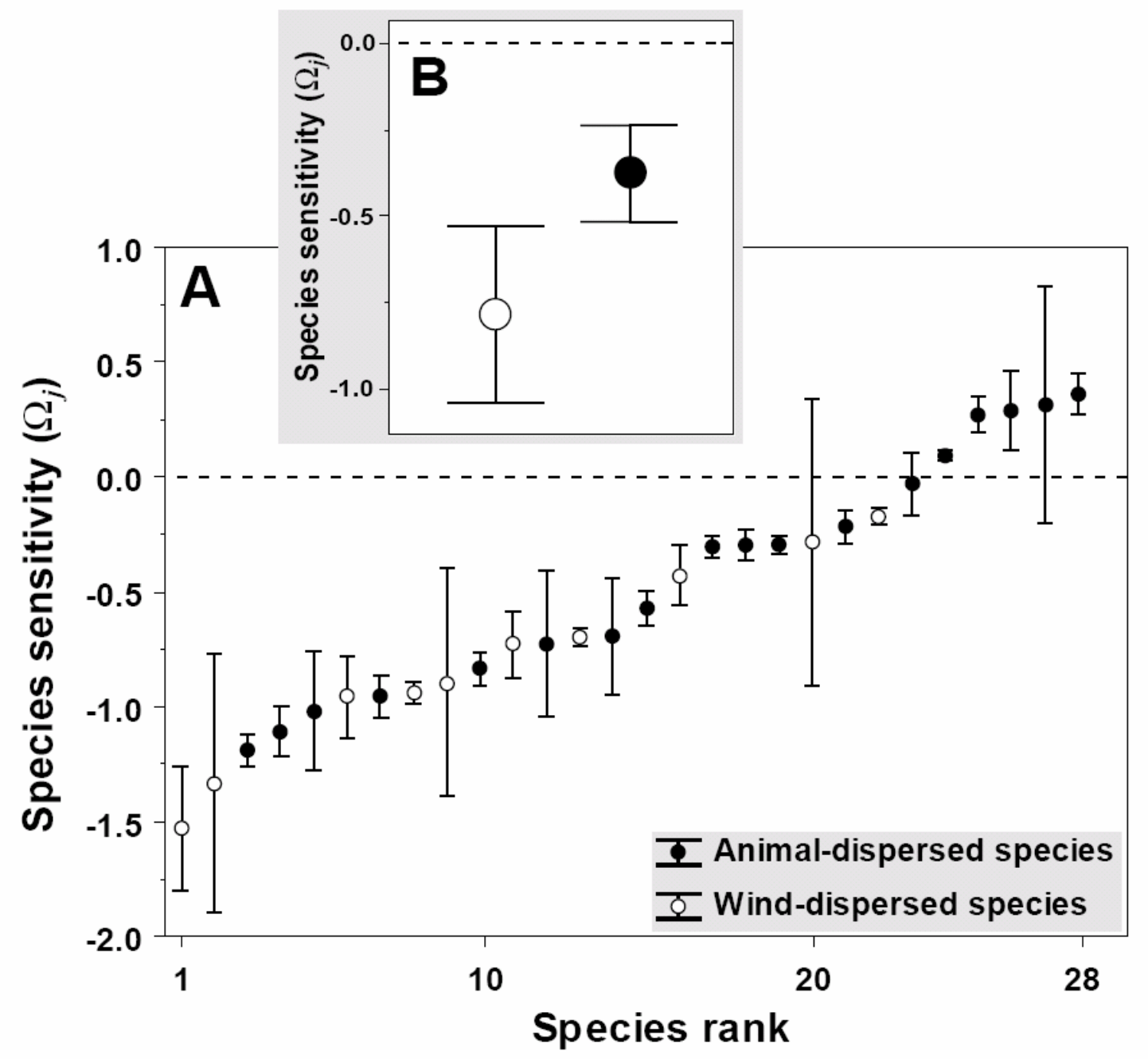


Figure S3. (A) Sensitivity of tree species to reductions in forest cover $\boldsymbol{\Omega}_{\mathrm{j}}$ after controlling for environmental factors. Results from permutation test for average difference between animal- and wind-dispersed species: $\mathrm{p}<0.05$. (B) Average $\Omega_{j}$ of wind-dispersed species $(n=12)$ and animal-dispersed species $(n=22)$ after controlling for environmental factors. Error bars are 95\% confidence intervals on the mean $\Omega_{j}$ for each group. Gray arrows indicate non-native species.

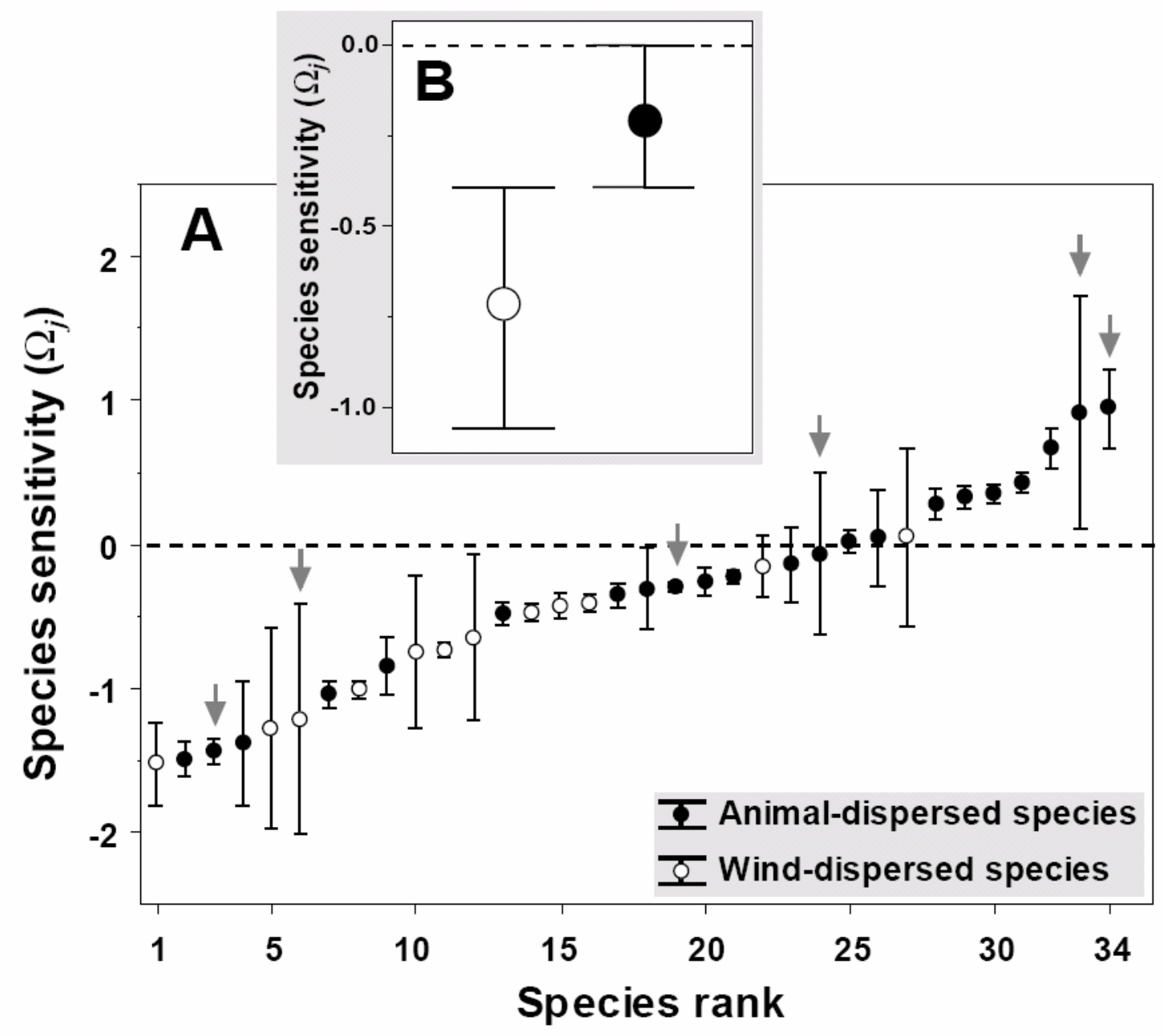

